\title{
CARCINOMA EPIDERMOIDE VARIANTE SARCOMATOIDE EM AMÍGDALA DIREITA - RELATO DE CASO
}

\section{ARTIGO ORIGINAL}

NETO, Alonso Alves Pereira ${ }^{1}$, ESPÓSITO, Mario Pinheiro², PELIZER, Carlos Antônio Albuquerque ${ }^{3}$, ESPÓSITO, Guilherme Soriano Pinheiro ${ }^{4}$, AMORIM, Leidiany Alves de ${ }^{5}$, BAZZO, Maria Luiza Veronese ${ }^{6}$, NETO, Raul Ivo Aureliano ${ }^{7}$

NETO, Alonso Alves Pereira. Et al. Carcinoma epidermoide variante Sarcomatoide em amígdala direita - relato de caso. Revista Científica Multidisciplinar Núcleo do Conhecimento. Ano. 06, Ed. 09, Vol. 07, pp. 139-150. Setembro 2021. ISSN: 2448-0959, Link de acesso: https://www.nucleodoconhecimento.com.br/saude/carcinoma-epidermoide, DOI: 10.32749/nucleodoconhecimento.com.br/saude/carcinoma-epidermoide

\section{RESUMO}

Neste relato de caso discutiremos o Carcinoma Epidermoide variante Sarcomatoide em amígdala. Desse modo, versaremos uma análise sobre a incidência, os tratamentos e as taxas de acometimento de tal doença. O objetivo é analisar a doença e procurar torná-la mais conhecida e popular, para que, caso algum médico se depare com ela, possa diagnosticar e tratar da forma mais eficiente, já que essa doença possui uma alta taxa de mortalidade. A cavidade oral e orofaríngea se

\footnotetext{
${ }^{1}$ Residente em Otorrinolaringologia e Cirurgia Cérvico-Facial, Graduado em Medicina pela União das Faculdades dos Grandes Lagos - UNILAGO.

${ }^{2}$ Doutor em Otorrinolaringologia pela Faculdade de Ciências Médicas da Santa Casa de São Paulo.

${ }^{3}$ Residente em Otorrinolaringologia e Cirurgia Cérvico-Facial; Graduado em Medicina pelo Centro Universitário São Lucas - UNISL.

${ }^{4}$ Residente em Otorrinolaringologia e Cirurgia Cérvico-Facial; Graduado em Medicina pelo Centro Universitário São Lucas - UNISL.

${ }^{5}$ Residente em Otorrinolaringologia e Cirurgia Cérvico-Facial; Graduada em Medicina pela Faculdade UMAX revalidado pela Universidade de Brasília - UNB.

${ }^{6}$ Residente em Otorrinolaringologia e Cirurgia Cérvico-Facial; Graduada em Medicina pela Universidade Iguaçu - UNIG.

${ }^{7}$ Residente em Otorrinolaringologia e Cirurgia Cérvico-Facial; Graduado em Medicina pelo Centro Universitário Maurício de Nassau - UNINASSAU.
}

RC: 98132

Disponível em: https://www.nucleodoconhecimento.com.br/saude/carcinomaepidermoide 
apresenta, sistematicamente, como um local capaz de alocar tumores. Há inúmeros fatores de risco, como: tabagismo, etilismo, imunodeficiência e má higiene bucal, tais fatores favorecem a malignização, principalmente em língua e assoalho bucal, portanto com uma investigação e intervenção precoce é possível curar e favorecer a sobrevida. Apresentaremos o caso de um paciente, que apesar de fatores de risco concordantes com literatura, apresentou tumor úlcero-infiltrativo orofaríngeo em amigdala direita e assimetria amigdaliana, com estudo histológico de carcinossarcoma ou carcinoma epidermóide sarcomatóide, sendo local incomumente acometido, com variante neoplásica rara, portanto, foi necessário questionar qual a melhor forma para tratar tal doença, tendo sido necessário uma discussão e estudo sobre as melhores formas de tratamento. Dessa forma, o paciente foi submetido radioterapia, quimioterapia e excisão cirúrgica por faringectomia direita parcial, sem linfadenectomia, pois felizmente não havia invasão angiolinfática. Apesar da rara localização, entende-se que a variante histológica é incomum e com isso sua evolução será pouco previsível. Com a intervenção cirúrgica adequada, associada a quimioterapia e radioterapia, há uma alta taxa de sobrevida prevista pela literatura, ao logo do acompanhamento do paciente foi possível perceber a eficiência da intervenção cirúrgica e uma evolução clínica significativa. Até o momento não há indícios clínicos e imagiológicos de recidiva da lesão.

Palavras-Chave: Sobrevida, Carcinoma Epidermoide Sarcomatóide, Excisão Cirúrgica, Intervenção Precoce, Tumor Úlcero-infiltrativo orofaríngeo.

\section{INTRODUÇÃO}

De acordo com Melo (2010), os tumores presentes na cabeça e no pescoço são apresentados pela grande relevância diante da alta taxa de mortalidade, portanto são considerados como problemas de saúde pública. Além do mais, a autora nos afirma que "Dentre os 6,4 milhões de neoplasias malignas diagnosticadas no mundo, aproximadamente $10 \%$ estão localizadas na boca, sendo esse o sexto tipo de 
câncer mais incidente no planeta." (MELO, 2010, n.p.), mostrando sua devida importância para a discussão.

Teixeira et al. (2009, p. 230), em seu estudo "Carcinoma Espinocelular da Cavidade Bucal: um Estudo Epidemiológico na Santa Casa de Misericórdia de Fortaleza" afirma que "o câncer de boca engloba um conjunto de neoplasias que acometem a cavidade bucal em suas mais variadas etiologias e aspectos histopatológicos.". A literatura procurou desenvolver um estudo acerca da porcentagem do câncer de boca, e percebeu-se que 90 a 95\% dos cânceres estão vinculados ao carcinoma espinocelular (CEC) ou epidermoide. Já o restante, 5 a 10\%, corresponde a sarcomas, neoplasias metastáticas, linfomas e leucemias, ou outras doenças mais raras.

Em 1864, Virchow descobriu o carcinossarcoma, descrito por ser um histológico incomum. O carcinossarcoma evidencia a natureza epitelial do componente sarcomatoide do carcinoma epidermoide de células fusiformes, sendo revelado por uma combinação de coloração imuno-histoquímica para queratinas. Esse tumor maligno também é conhecido como carcinoma pleomórfico, carcinoma polipoide, carcinoma sarcomatoide, pseudossarcoma, carcinossarcoma ou carcinoma metaplásico. De acordo com Morais et al. (2014), o carcinossarcoma "apesar de poder apresentar-se em qualquer parte do corpo, cabeça e pescoço são os locais de maior prevalência. A maioria dos casos surge na cavidade oral, na laringe, nas amígdalas e na faringe.".

A literatura conseguiu perceber que as áreas mais acometidas eram: as regiões dos lábios, a cavidade oral (mucosa bucal, gengivas, palato duro, língua e assoalho), a orofaringe (amígdalas, palato mole e base de língua), a glândulas salivares, o trígono retromolar, a valécula, a loja tonsilar, as tonsilas palatinas e as paredes posterior e laterais da orofaringe, como nos afirma Silva; Leão e Scarpel (2009). Os autores ainda observaram que a língua e lábio inferior eram localizações anatomicamente mais comuns para que o Carcinoma de Células Escamosas (CCE) se apresentasse primeiro.

RC: 98132

Disponível em: https://www.nucleodoconhecimento.com.br/saude/carcinomaepidermoide 
Os estudos também descobriram uma necessidade de descobrir quem eram os mais afetados por essas neoplasias, portanto foi verificado que os indivíduos do sexo masculino com idade superior a 50 anos, etilistas, fumantes e com um mal estado de conservação e higiene buco-dentário eram os mais acometidos pela doença. A literatura relata que o álcool e o tabaco são fatores de risco para que o CCE oral ocorra. Entende-se que não há um agente definido para causar o CCE isoladamente, porém os fatores extrínsecos e intrínsecos quando em atividade podem causar a doença. É importante salientar que a hereditariedade, para a literatura, não desempenha um papel etiológico importante no carcinoma oral.

Os fatores extrínsecos ou ambientais são fatores importantes para o desenvolvimento do CCE e estão envolvidos: o tabagismo, o etilismo, as dietas pobres em vegetais, a exposição à luz ultravioleta, a infecção viral, a má-higiene bucal; já os fatores intrínsecos são referentes ao indivíduo: a idade, a raça, o sexo, as mutações espontâneas e/ou herdadas. A literatura compreende que a prevalência da doença se estabelece na ação de agentes físicos e químicos com potencial mutagênico, mas entende-se que quando combinado tabaco e consumo de bebidas alcoólicas a causa das neoplasias bucais é de $65 \%$ a $90 \%$.

Teixeira et al. (2009, p. 230) ainda nos afirma que o "papilomavírus humano (HPV) também pode comportar-se como mais um cocarcinógeno para o câncer de cavidade oral, assim como a radiação solar para carcinomas de lábio.". Ao obtermos o conhecimento dos fatores de risco pode-se construir uma prevenção efetiva para a doença, bem como reconhecer a sintomatologia por intermédio do paciente. Dessa forma, o diagnóstico será realizado precocemente e o indivíduo poderá ser encaminhado imediatamente para tratamento, o que auxilia na redução da morbidade e mortalidade causadas pelo câncer. De acordo com Melo (2010) "o diagnóstico precoce é fator preponderante nas taxas de sobrevida que podem atingir $70 \%$ a $90 \%$, quando as lesões ainda são pequenas e localizadas", mostrando a necessidade do diagnóstico para que se tenha um tratamento melhor. 
Embora a boca permita um acesso fácil ao exame visual, os carcinomas em geral não são fáceis de diagnosticar quando assintomáticos. De acordo com Dedivitis et al. $(2004$, p. 36$)$,

O câncer da orofaringe tem como agravante ser oligossintomático no início, seja devido ao padrão de inervação sensitiva, dada pelo glossofaríngeo, seja devido à superfície irregular da mucosa, em especial a das tonsilas palatinas, com suas criptas, onde um pequeno carcinoma pode ficar oculto ao exame clínico, ou ainda pelo padrão de inspeção do paciente, nem sempre muito atento.

De acordo com Venturi; Pamplona; Cardoso (2004) o CCE da cavidade oral, no estágio inicial mostra-se distinto, clinicamente. Pode até mesmo ser apresentado através de lesões: leucoplásica, eritroplásica, leucoeritroplásica, ou úlceras que não conseguem cicatrizar. Considera-se que a assimetria amigdalina seja suspeita de malignidade, porém pode ser apenas um achado incidental quando ausente de outros fatores de risco, nestes casos, é apresentado com indício de malignidade.

Gonçalves et al. (2020, p. 96) afirmam que "uma das indicações para amigdalectomia é a suspeita de malignidade". É necessário compreender que os riscos de metástase estão correlatos ao estadiamento dos linfonodos e não com o tamanho do tumor primário. Portanto, quanto mais próximo da linha média, maior o risco de propagação para linfonodos de ambos os lados da região cervical. É fundamental que se faça um exame físico cuidadoso na boca para que se identifique as lesões precursoras e/ou iniciais da patologia, mas é imprescritível a necessidade dos exames complementares, como a biópsia, para que se conclua o diagnóstico.

De acordo com Volkweis et al. (2014, p. 64) "No Brasil o índice de identificação de lesões malignas iniciais na boca é muito baixo, correspondendo a menos de $10 \%$ dos casos diagnosticados". Nos é muito cara as informações do registro hospitalar de câncer do Hospital do Câncer I ( $\mathrm{HCl}$ ) do INCA, pois demonstra-nos que $70 \%$ dos pacientes atendidos com diagnóstico de câncer de boca encontravam-se em estágios avançados, o que prejudica seriamente a sobrevida. Devido a isso, a mortalidade por meio do câncer da cavidade oral ocupa o nono lugar entre os outros 
tumores malignos, correspondendo a 2,8\% das mortes por câncer no Brasil, afirma Teixeira et al. (2009).

O tratamento para o câncer bucal depende do local, do estágio, dos tratamentos prévios, da histopatologia e da idade do paciente. Pode ser necessário uma intervenção cirúrgica, ou radioterapia e, a depender do tratamento o uso da quimioterapia, porém há casos em que será necessário a associação entre eles. Dependendo das áreas acometidas e do procedimento cirúrgico, os pacientes podem apresentar alterações orgânicas e funcionais, como: dificuldades na mobilidade, desempenho das estruturas e funções orofaciais (fonoarticulação, voz e deglutição), como nos mostra a literatura.

De acordo com Moro et al. (2018, n.p.)

As taxas de sobrevida em 5 anos dos carcinomas oral e orofaríngeo são aproximadamente $50 \%$, e a maioria desses pacientes sobrevivem pouco tempo após o diagnóstico. Isso se deve ao fato de que a maioria dos tumores é identificada tardiamente, comprometendo o tratamento, o prognóstico e a sobrevida dos pacientes. Por isso, a divulgação de informações e dados estatísticos sobre o câncer de boca e orofaringe é necessária, para estimular os profissionais a realizarem ações para detecção precoce, contribuir para um melhor entendimento da doença e de propostas terapêuticas mais viáveis, otimizando, consequentemente, as taxas de sobrevida.

As recomendações nacionais de prevenção é que se tenha uma higiene oral melhor e visitas regulares aos dentistas. Além do mais, é fundamental um exame clínico cuidadoso da boca. Já para os indivíduos de maior risco (tabagistas e etilistas), o exame da boca deve ser sistemático; já para os indivíduos que apresentam lesões suspeitas o encaminhamento imediato deve ser feito para que se realize os procedimentos diagnósticos necessários.

\section{RELATO DE CASO}

Paciente R. C. S. 69 anos, sexo masculino, foi encaminhado ao consultório em julho de 2020 para avaliação de quadro de disfagia e odinofagia progressiva há 1 mês, sem dispneia ou disfonia. No exame físico - oroscopia, cavidade oral com presença

RC: 98132

Disponível em: https://www.nucleodoconhecimento.com.br/saude/carcinomaepidermoide 
de lesão nodular vegetante em amigdala direita na orofaringe, uma lesão palpável e indolor, evidenciado assimetria de amigdala sendo maior a direita que a esquerda. Ausência de linfonodomegalia palpável em região cervical. O paciente relata tabagismo por 25 anos e parou há 20 anos e nega etilismo atual, parou há 15 anos. É hipertenso em uso Losartana 50mg 12/12h e diabético em uso Metformina 850mg dia e Glibenclamida 5mg dia. Nega outras comorbidades.

Fig.1: Lesão nodular pediculada em Amígdala direita
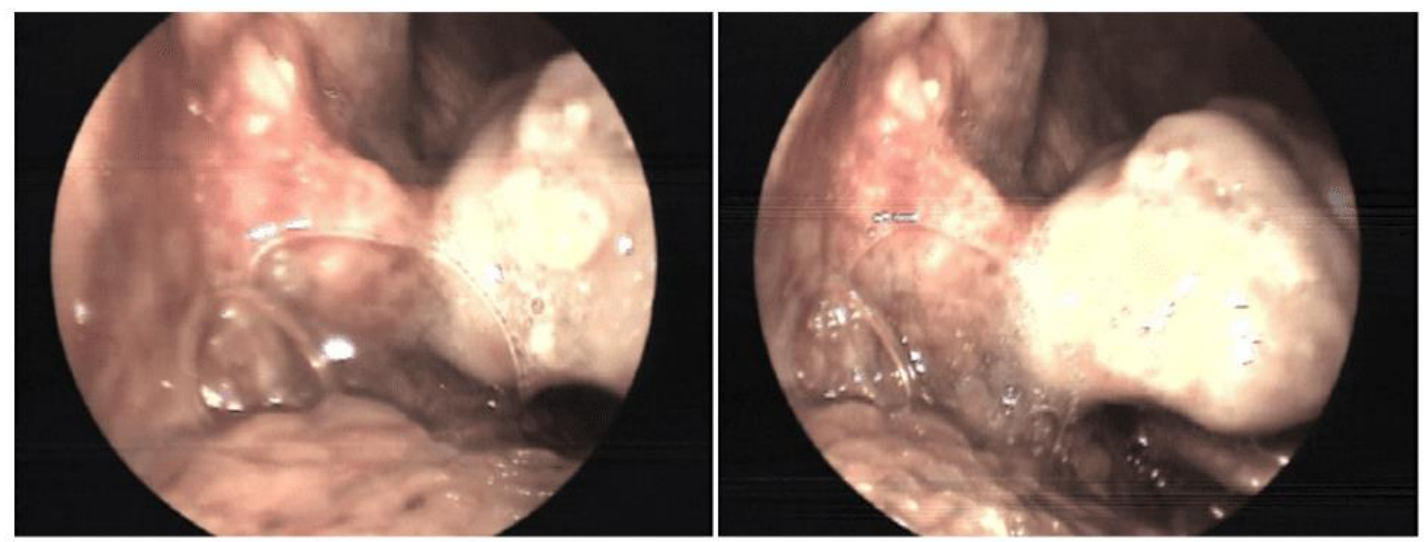

Fonte: Autor.

O exame de videolaringoscopia (VDL) evidenciou uma lesão nodular vegetante pediculada em amigdala direita de $2,5 \mathrm{~cm}$ de diâmetro. TC de pescoço com formação expansiva solida polipoide em orofaringe parede lateral direita, de contornos lobulados e limites definidos, discreto realce pós contraste, linfonodos infracentimetricos em cadeias cervicais superiores e regiões submandibulares. Após conclusão da biopsia, o anatomopatológico revelou lesão vegetante de orofaringe direita como neoplasia maligna invasiva sugestiva de carcionoma epidermoide variante sarcomatoide de células fusiformes. Logo, realizou-se os pedidos de exames pré-operatórios.

O paciente foi submetido a Faringectomia parcial direita sem linfadenectomia, em 06 de agosto de 2020, com anatomopatológico de Carcionoma Epidermoide moderadamente diferenciado (60\%), com áreas pouco diferenciadas (sarcomatoides 
$30 \%$ ), infiltração perineural presente, ausência de invasão angiolinfatica, margens cirúrgicas livres, de 4,0 × 2,5 × 1,5 cm. Houve a exérese completa da lesão, com margens livres e o paciente foi encaminhado para a Oncologia Clínica onde foi submetido a Quimioterapia e Radioterapia no período 17 de novembro de 2020 a 06 de janeiro de 2021. Até o momento, junho de 2021, apresenta cicatriz em bom estado, assintomático e sem indícios clínicos e imagiológicos de recidiva da lesão.

\section{DISCUSSÃO}

Neste artigo, relata-se um caso clínico e uma revisão sistemática sobre Carcinoma Epidermoide Variante Sarcomatoide de células fusiformes em regiões de cabeça e pescoço. Referido anteriormente, a literatura compreende que um dos aspectos epidemiológicos do câncer de boca e orofaringe, é o predomínio no sexo masculino, condição atuante em nosso estudo de caso. Outro aspecto epidemiológico que está em consonância com nosso estudo de caso é a idade preferencial, de 50 aos 70 anos.

Alguns estudiosos afirmam que os tumores estão alocados nas regiões da língua e do assoalho oral, localidade esta que prevalece o câncer de boca, entretanto em nosso estudo o local acometido foi a tonsila palatina direita em orofaringe. A relação estabelecida entre o etilismo e o tabagismo no desenvolvimento do carcinoma epidermóide de cabeça e pescoço já está bem desenvolvido, tais fatores podem ser encontrados em nosso estudo de caso, visto que o paciente relata o uso de tabaco por 25 anos e era, também, etilista.

De acordo com Carli et al. (2009, p. 209),

A maioria dos estudos sobre a prevalência de câncer bucal ressalta a importância que a localização do tumor exerce sobre o prognóstico dos pacientes acometidos. A vascularização sanguínea e linfática nas diferentes localizações anatômicas pode influenciar tanto a evolução tumoral quanto a sobrevida dos pacientes. Tumores localizados em língua e assoalho bucal podem mais facilmente disseminar ocasionando o desenvolvimento de metástases regionais, enquanto lesões situadas em gengiva e lábio mais raramente estão associadas a metástases.

RC: 98132

Disponível em: https://www.nucleodoconhecimento.com.br/saude/carcinomaepidermoide 
É importante constar que em nosso estudo o local acometido foi a parede lateral direita de orofaringe, em amigdala direita e não houve metástases, sem acometimento de linfonodos cervicais. O paciente em estudo apresentou uma lesão nodular vegetante em parede lateral direita de orofaringe, uma lesão palpável e indolor ao exame físico da orofaringe, ao contrário do descrito, não havia áreas de ulceração ou aspecto infiltrativo da lesão. De acordo com Souza; Sakae; Guedes (2008, p. 38) compreende que "a apresentação macroscópica de lesão úlceroinfiltrativa no câncer de boca e a lesão nodular-submucosa com pequena área ulcerada no câncer de orofaringe como os aspectos mais comuns com os quais essas doenças se apresentam".

Para que o diagnóstico seja eficiente é necessário observar se houve carcinoma espinocelular, os sintomas mais recorrentes da prevalência de câncer de boca e orofaringe é a "ferida na boca" e a odinofagia. Em nosso estudo, o paciente estava em estágio inicial, o que o difere é o histológico incomum: carcionoma epidermoide variante sarcomatoide sem aspecto úlcero-infiltrativo; o principal sintoma referido foi odinofagia seguido de disfagia.

A literatura afirma que o diagnóstico do carcinoma de orofaringe, em sua maioria, é feito tardiamente, as causas do tardio diagnóstico se dão devido à ausência de sintomatologia precoce, além do mais, percebe-se a falta de preparo dos profissionais (médicos e dentistas) e a sintomatologia pobre. Em nosso estudo obtivemos um diagnóstico precoce, já que o paciente apresentou menos de um mês de sintomas. O tratamento foi iniciado de imediato após confirmação com anatomopatológico.

A literatura relata o carcinoma epidermoide de células fusiformes como uma variante agressiva do carcinoma escamoso, sendo mais propensa a espalhar-se para linfonodos regionais e sítios à distância e possui maior recidiva local. Em nosso estudo mesmo com uma variante histológica incomum foi realizado diagnóstico precoce, deste modo não houve invasão de estruturas adjacentes e sem acometimento de linfonodos cervicais. 
Há uma relação significativa entre assimetria amigdalina e doença maligna, com a presença de um fator de risco há a suspeita de malignidade, podendo não ser apenas a idade, outros exemplos são: história prévia de neoplasia, lesões objetiváveis da amígdala, alteração da consistência amigdalina, adenopatia cervical concomitante, tabagismo, sintomas constitucionais, estados de imunossupressão ou um rápido crescimento amigdalino unilateral. Em nosso caso, por se tratar de neoplasia maligna em amigdala direita, foi realizada a faringectomia parcial direita.

Percebemos, que o melhor tratamento para os indivíduos com neoplasia em orofaringe, com anatomopatológico carcinoma epidermóide, se dá através da combinação de radioterapia e quimioterapia e em seguida de excisão cirúrgica completa da neoplasia, margens livres. No estudo de caso a conduta foi a mesma do tratamento de escolha citado na literatura.

É necessário que haja um conhecimento dos médicos e dos dentistas acerca das características clínicas e epidemiológicas de pacientes com câncer de boca e orofaringe, com indicação ou não de tratamento cirúrgico, para que assim tenhamos um tratamento mais eficaz, diminuindo, dessa forma, a mortalidade. $O$ aumento da ocorrência de carcinomas orais está relacionado aos métodos mais avançados de diagnóstico, aumento do número de estomatologistas e patologistas orais. Há mais núcleos de referência em diagnóstico, informatização dos centros de referência, uma preocupação maior com a compilação de dados e anotações nos prontuários. Com a globalização elevasse o acesso dos dados mundiais, está havendo uma publicação e uma disseminação de informação científica, aumentando assim o número de campanhas informativas.

\section{CONSIDERAÇÕES FINAIS}

Interpretando as referências literárias, o caso apresentado, foi concordante com a incidência etária e de gênero, sendo o paciente do sexo masculino e situado na faixa de idade entre 50 a 70 anos. Alguns fatores de risco imperativos em literatura para malignização da lesão em cavidade oral e orofaríngea, como tabagismo e etilismo, 
também estavam presentes nos hábitos do paciente em questão. Em contradição, com relação aos sítios anatômicos mais acometidos, a tonsila palatina em orofaringe é um local diagnóstico incomum, sendo a incidência da língua e assoalho de boca os locais mais frequentes. A conduta terapêutica utilizada foi a combinação entre radioterapia, quimioterapia e excisão cirúrgica completa da neoplasia, como orientado em referências bibliográficas. $O$ paciente fora mantido sob acompanhamento clínico e cirúrgico rigoroso, não possui recidiva tumoral, até o momento. O reconhecimento da lesão através do exame físico da cavidade bucal e dos exames complementares no atendimento inicial é fundamental para intervenção precoce nos estágios iniciais do câncer oral e de orofaringe, o que resulta em um correto tratamento e bom prognóstico ao paciente, mesmo que seja uma variante neoplásica rara.

\section{REFERÊNCIAS}

CARLI, Marina; SANTOS, Sophia; PEREIRA, Alessandro; HANEMANN, João Adolfo. Características Clínicas, Epidemiológicas e Microscópicas do Câncer Bucal Diagnosticado na Universidade Federal de Alfenas. Revista Brasileira de Cancerologia, v. 55, n. 3, p.205-211, 2009. Disponível em: < http://www1.inca.gov.br/rbc/n_55/v03/pdf/09_artigo1.pdf>. Acesso em: 05/07/2021.

DEDIVITIS, Rogério; FRANÇA, Cristiane; MAFRA, Ana Claudia; GUIMARAES, Fernanda; GUIMARAES, André. Características clínicoepidemiológicas no carcinoma espinocelular de boca e orofaringe. Rev Bras Otorrinolaringol., v.70, n.1, p. 2004. 35-40, Disponível em: < https://www.scielo.br/j/rboto/a/gfwsFyNsKWKCLZDMrqYBQTm/?lang=pt\&format=pdf >. Acesso em: 05/07/2021.

GONÇALVES, Ana Isabel; PEREIRA, Diogo; DUARTE, Delfim; LOPES, Gustavo; VILHENA, Ditza. Assimetria das amígdalas palatinas - Resultados do Serviço de Otorrinolaringologia de um Hospital Terciário. Revista Portuguesa de Otorrinolaringologia e Cirurgia de Cabeça e Pescoço, v. 57, n.3, 2019. Disponível 
em: < https://www.journalsporl.com/index.php/sporl/article/view/787/660 >. Acesso em: 05/07/2021.

MORAIS, Everton; MACEDO, Rômulo; REGES, Diogo; MORAIS, Maria de Lourdes. Carcinoma epidermoide de células fusiformes de cabeça e pescoço: revisão sistemática. Brasília Med, v. 51, n. 2, p. 151-158, 2014. Disponível em: < https:/ /cdn.publisher.gn1.link/rbm.org.br/pdf/v51n2a11.pdf> Acesso em: 05/07/2021.

MELO, Letícia; SILVA, Marcelle; BERNARDO, Joyce Maria; MARQUES, Eduardo; LEITE, Isabel Cristina. Perfil epidemiológico de casos incidentes de câncer de boca e faringe. RGO - Rev. Gaúcha Odontol., v. 58, n. 3, p. 351-355, 2010. Disponível em:

http://revodonto.bvsalud.org/scielo.php?script=sci_abstract\&pid=S1981-

86372010000300012\&lng=en\&nrm=iso\&tlng=pt\#: :text=RESULTADOS\%3A\%20Dos \%20101\%20pacientes\%2C\%2084, inferior\%20\%C3\%A0\%20m\%C3\%A9dia\%20do\% 20grupo. >. Acesso em: 05/07/2021.

MORO, Juliana; MARONEZE, Marília; ARDENGHI, Thiago; BARIN, Luisa; DANSI, Cristiane. Câncer de boca e orofaringe: epidemiologia e análise da sobrevida. Einstein, v. 16, n. 2, p. 1-5, 2018. Disponível em: < https://www.scielo.br/j/eins/a/jKmLSDsfpRhQLx4rfNr6mNx/?lang=pt\#: :text=As\%20t axas\%20de\%20sobrevida\%20em\%205\%20anos\%20dos\%20carcinomas\%20oral,po uco\%20tempo\%20ap\%C3\%B3s\%200\%20diagn\%C3\%B3stico.\&text=Isso\%20se\%20 deve\%20ao\%20fato,e\%20a\%20sobrevida\%20dos\%20pacientes.>. Acesso em: 05/07/2021.

REZENDE, Caio Perrella; RAMOS, Marcelo Barboza; DAGUILA, Carlos Henrique; DEDIVITIS, Rogério Aparecido; RAPOPORT, Abrão. Alterações da saúde bucal em portadores de câncer da boca e orofaringe. Rev. Brasileira Otorrinolaringologia, v.74, n.4, p. 596-600, 2008. Disponível em: < https://www.scielo.br/j/rboto/a/7smpPzSfJ57T6LZTSRnBBgx/?lang=pt\#: :text=CONC LUS\%C3\%83O- 
,Os\%20resultados\%20permitem\%20concluir\%20pela\%20presen\%C3\%A7a\%20de\% 20associa\%C3\%A7\%C3\%A30\%20de\%20doen\%C3\%A7a,de\%20higiene\%20ou\%20 condi\%C3\%A7\%C3\%A30\%20dent\%C3\%A1ria.>. Acesso em: 05/07/2021.

SILVA, Patrícia; LEAO, Verônica; SCARPEL, Renata. Caracterização da população portadora de câncer de boca e orofaringe atendida no setor de cabeça e pescoço em hospital de referência na cidade de Salvador - BA. Rev. CEFAC, v.11, Supl. 3, p. 441-447, 2009. Disponível em: < https://www.scielo.br/j/rcefac/a/vWVjsLJWRLKn4wRwbzccDmd/abstract/?lang=pt>. Acesso em: 05/07/2021.

SOUZA, Rafael; SAKAE, Thiago; GUEDES, André. Características clínicoepidemiológicas de pacientes portadores de carcinomas da cavidade oral e orofaringe em clínica privada no sul do Brasil. Arquivos Catarinenses de Medicina, v. $37, \quad$ n. 2,2008 . Disponível em: < https://pesquisa.bvsalud.org/portal/resource/pt/lil-499747>. Acesso em: 05/07/2021.

TEIXEIRA, Ana Karine; ALMEIDA, Maria Eneide; HOLANDA Marcelo; SOUSA, Fabrício; ALMEIDA, Paulo César. Carcinoma Espinocelular da Cavidade Bucal: um Estudo Epidemiológico na Santa Casa de Misericórdia de Fortaleza. Revista Brasileira de Cancerologia, v.55, n.3, p. 229-236, 2009. Disponível em: < http://www1.inca.gov.br/rbc/n_55/v03/pdf/33_artigo4.pdf>. Acesso em: 05/07/2021.

VENTURI, Beatriz; PAMPLONA, Ana; CARDOSO, Abel. Carcinoma de células escamosas da cavidade oral em pacientes jovens e sua crescente incidência: revisão de literatura. Rev Bras Otorrinolaringol., v.70, n.5, p. 679-86, 2004. Disponível em: <https://www.scielo.br/j/rboto/a/K8fXCWTwzLvv8rZnRr8Wrjj/?lang=pt>. Acesso em: 05/07/2021.

VOLKWEIS, Maurcio; BLOIS, Matheus; ZANIN, Roberto, ZAMBONI, Rodrigo. Perfil Epidemiológico dos Pacientes com Câncer Bucal em um CEO. Rev. Cir. Traumatol. Buco-Maxilo-Fac., v.14, n.2, p. 63-70, 2014. Disponível em: < RC: 98132

Disponível em: https://www.nucleodoconhecimento.com.br/saude/carcinomaepidermoide 
https://www.revistacirurgiabmf.com/2014/2/brjoms.14.2.10.pdf>

Acesso

em: 05/07/2021.

Enviado: Junho, 2021.

Aprovado: Setembro, 2021. 